\title{
Random matrices and the replica method
}

\author{
E. Kanzieper \\ The Abdus Salam International Centre for Theoretical Physics, P. O. Box 586, 34100 Trieste, Italy
}

(29 August 1999)

\begin{abstract}
Recent developments [Kamenev and Mézard, cond-mat/9901110, cond-mat/9903001; Yurkevich and Lerner, cond-mat/9903025; Zirnbauer, cond-mat/9903338 have revived a discussion about applicability of the replica approach to description of spectral fluctuations in the context of random matrix theory and beyond. The present paper, concentrating on invariant non-Gaussian random matrix ensembles with orthogonal, unitary and symplectic symmetries, aims to demonstrate that both the bosonic and the fermionic replicas are capable of reproducing nonperturbative fluctuation formulas for spectral correlation functions in entire energy scale, including the self-correlation of energy levels, provided no $\sigma$-model mapping is used.
\end{abstract}

PACS number(s): 05.40.-a, 05.45.Mt

\section{INTRODUCTION}

Energy level statistics of electrons in a random potential is one of the basic issues in the theory of disordered conductors. It is also a classic example of the problem whose proper statistical description calls for a use of nonperturbative methods of averaging over disorder realizations. The latter procedure is not trivial because the spectral observables depend in highly nonlinear fashion on a random Hamiltonian, thus making the calculation of the ensemble averages very difficult. Within the single electron picture, it is necessary to average a product of resolvents, $\left(\mathbf{r}\left|(z-\mathcal{H})^{-1}\right| \mathbf{r}^{\prime}\right)$, that admits an exact representation through a ratio of two functional integrals over auxiliary fields which may consist of either commuting [bosonic] or anticommuting [fermionic] entries. This representation, however, is still not convenient for nonperturbative averaging due to an awkward denominator. To get rid of it, Edwards and Andersont proposed a replica method that substitutes the initial disordered system by the $n$ identical noninteracting systems. Such a trick removes a randomness from the denominator and, therefore, makes it possible to perform a nonperturba- tive averaging over the random potential from the very beginning for all integer $n$ which must be set to zero at the appropriate stage [by the analytic continuation $n \rightarrow 0$ ]. Depending on the origin of the auxiliary fields, the resulting effective field theory is defined on either a noncompact 2 or a compact 1 manifold.

An alternative approach was invented by Efetov $\$$ who introduced an auxiliary field with an equal number of bosonic and fermionic entries that obviates the need for replicas. This supersymmetry formalism turned out to be very fruitful in the theory of disordered conductors and beyond 5 . Along with other fundamental results, it has led to establishing a link 1 between the theory of disordered metals and the random matrix theory [RMT] founded by Wignerl, thus confirming on microscopic grounds a much earlier conjecture by Gorkov and Eliashberg]. To be precise, it was demonstrated that the 'density-density' correlation function $R_{\beta}(s)$ of disordered conductor in the ergodic limit is solely determined by the global symmetries of the system and coincides with the RMT predictions. For the three symmetry classes - orthogonal $(\beta=1)$, unitary $(\beta=2)$, and symplectic $(\beta=4)$ - the 'density-density' correlation functions are

$$
\begin{aligned}
& R_{1}(s)=1-\frac{\sin ^{2}(\pi s)}{(\pi s)^{2}}-\int_{s}^{+\infty} d t \frac{\sin (\pi t)}{\pi t} \frac{d}{d s}\left(\frac{\sin (\pi s)}{\pi s}\right)+\delta(s), \\
& R_{2}(s)=1-\frac{\sin ^{2}(\pi s)}{(\pi s)^{2}}+\delta(s), \\
& R_{4}(s)=1-\frac{\sin ^{2}(2 \pi s)}{(2 \pi s)^{2}}+\int_{0}^{s} d t \frac{\sin (2 \pi t)}{2 \pi t} \frac{d}{d s}\left(\frac{\sin (2 \pi s)}{2 \pi s}\right)+\delta(s),
\end{aligned}
$$

$s$ being the distance between the eigenlevels measured in units of the mean level spacing $\Delta$. The range of validity of Eqs. (1.1) is $s \ll g$, where $g \gg 1$ is the dimensionless Thouless conductance.

While the success 5 of the supersymmetry approach has been yery impressive, the alternative replicated field theories2 3 [which, besides, are suitable for a treatment of disordered systems in presence of interactions?] seemed to be restricted 10 to reproducing the perturbative diagrammatic expansions 11 only. A detailed analysis of this problem has been given by Verbaarschot and Zirnbauer in Ref 12 , where the failure of the replica method [in its $\sigma$-model formulation] to correctly account for all nonperturbative contributions has been attributed to the fact 
that the replica trick is mathematically ill founded, and the knowledge of the replicated partition function for all integer $n$ need not be sufficient for extrapolation to the limit $n \rightarrow 0$ which, generically, can also be nonunique 13 .

Surprisingly enough, in the very recent publication 14 , Kamenev and Mézard have formulated a variant of the fermionic replica method that allowed them to rederive Eq. $1.1 \mathrm{~b}$, $s \gg 1$, in the context of RMT for the Gaussian Unitary Ensemble [GUE]. The approach 14 has relied on the combination of the exact integration over the angular degrees of freedom of the replicated $\sigma$-model, by using the Itzykson-Zuber integral15, and the approximate saddle point calculations in the $n$-dimensional space of remained eigenvalues $\hat{\Lambda}=\operatorname{diag}\left(\lambda_{1}, \ldots, \lambda_{n}\right)$. In order to correctly reproduce the result Eq. (1.1b) for GUE in the large $-s$ limit, one is forced to take into account the nontrivial saddle points which break the replica symmetry between the different eigenvalues $\lambda_{i}, 1 \leq i \leq n$. On the formal level, this implies that the $n$-component vector of eigenvalues ascribes a nontrivial internal structure which must be kept in the limit $n \rightarrow 0$ of vanishing number of integration variables! Very similar structure of the vector saddle peint manifolds is present in two subseqment publications 16.17 that extended the results of Ref.14 to two other [orthogonal ap symplectic] symmetry classes both in the RMT limit17 and beyond it 16 . A cal discussion of the prefedures employed in Refs.14.16, 17 has been given in Ref. 19 where it has been shown that there exists some additional input in the calculational schemes 14 16.17 which is equivalent to introducing the causality information. A conjecture was mut forward 19 that the fermionic replicas in elaboration 14 . 16.17 are perturbatively equivalent to the supersymmetry method with $s^{-1}$ being a cmall parameter. It was also explained 9 that the result.14. 17 are 'semiclassically exact' for the unitary symmetry class owing to the Duistermaat-Heckman theoremen.

There is one common feature inherent to the above mentioned replicated calculations: All of them, starting with either bosonic or fermionic replicas, reduce the problem of evaluating the spectral density-density correlation function to the effective $\sigma$-model defined in the configurational space of replicas only. Correspondingly, the 'coordinate' space disappears from the theory, and the memory of the initial [matrix] Hamiltonian is only retained in the symmetry of the auxiliary $\hat{Q}$-fields. An immediate consequence of such a treatment is that the integral representations for the spectral observables appear to involve the integration measures which become senseless as $n \rightarrow 0$. This can be seen, for instance, from the Verbaarschot-Zirnbauerintegral for the two-level correlation function derived ${ }^{2} 2$ from the fermionic replicas for the GUE in the large $-N$ limit:

$$
R(r)=-\lim _{n \rightarrow 0} \frac{1}{n^{2}} \partial_{r}^{2} \int_{-1}^{+1} \prod_{m=1}^{n}\left(d \lambda_{m} \mathrm{e}^{i r \lambda_{m}}\right)
$$

$$
\times \prod_{1 \leq m_{1}<m_{2} \leq n}\left|\lambda_{m_{1}}-\lambda_{m_{2}}\right|^{2} .
$$

[Here, $r=\pi s$ in notations of Eq. (1.1b)]. Obviously, one cannot simply take the limit $n \rightarrow 0$ neither in the above integral representation nor in the result of the integration

$$
R(r)=-\lim _{n \rightarrow 0} \frac{1}{n^{2}} \partial_{r}^{2} \operatorname{det}\left(\partial_{r}^{i+j-2} \frac{\sin r}{r}\right)_{1 \leq i, j \leq n}
$$

because of the vanishing dimensionality of the matrix under the sign of the determinant. Therefore, one should know how to evaluate the integrals of the type Eq. (1.2) in the closed form for arbitrary integer $n$ in order to analytically continue the resulting expression from integer $n$ and perform the replica_limit $n \rightarrow 0$ afterward. One such way proposed in Ref.17 reduces Eq. (1.2), in the domain $r \gg 1$, to the expression which coincides identically with the one obtained by means of the 'replica symmetry breaking' [RSB]. This coincidence signals that the RSB may be viewed as a useful language for approximate computation of the integrals defined on the problematic [in the above mentioned sense] measures. In the course of exact calculations, the notion of RSB must not appear at all.

The studies 12 14, 26. 75.19 call for further clarification of the question that has a conceptual importance and concerns the applicability of the replica method to a nonperturbative description of the eigenlevel correlations in general, and those on the short distance scale, in particular. The central issue to be learnt here is whether the replica method itself faces some internal insurmountable obstacles, or existing difficulties are to be attributed to the particular computational schemes. We believe that in order to answer this question, one has to depart from conventional routes toward exact calculational schemes within the replica framework.

While it is unknown so far how the exact replica treatment could be realized for realistic disordered systems, the present paper aims to demonstrate that there is one particular but conceptually important situation where the exact computation is possible for both the fermionic and the bosonic replicas. This is the case of invariant random matrix ensembles 21 with a general non-Gaussian measure

$$
P_{N}[\mathbf{H}] \propto e^{-\beta \operatorname{tr} V[\mathbf{H}]}
$$

specified by the confinement potential $V[\mathbf{H}]$ and the Dyson index $\beta=1,2$ and 4 that labels the symmetry of the matrix Hamiltonian $\mathbf{H}$.

A distinctive feature of our treatment that must be emphasized from the very beginning is that we do not use a $\sigma$-model representation for the replicated partition function averaged over the ensemble Eq. (1.4) of random matrices. Instead, a different technique is used for exact averaging of the product statistics that comprises the replicated partition function as a particular case. This technique generates especially useful representations for the spectral observables, thus allowing one 
to avoid the problem of the ill defined integration measure. As a direct consequence of that, we construct [in a simple and straightforward way] a well defined replica limit $n \rightarrow 0$ in expressions for the spectral observables that turn out to identically coincide with Eqs. (11.1). This coincidence, observed for arbitrary $s$, is the main outcome of the study.

The following program is carried out:

(i) First, the replicated partition function of the form

$$
\begin{aligned}
Z_{\gamma, \gamma^{\prime}}^{n}\left(E, E^{\prime}\right) & =\operatorname{det}^{n}\left[(E-\mathbf{H})^{2}+\gamma^{2}\right] \\
& \times \operatorname{det}^{n}\left[\left(E^{\prime}-\mathbf{H}\right)^{2}+\gamma^{\prime 2}\right]
\end{aligned}
$$

is introduced in accordance with the very concept of the replica method that boils down to constructing such a generating function [e. g., for the 'density-density' correlator] which does not contain a random denominator at the expense of the artificial appearance of replica parameter $n$ in Eq. (1.5).

(ii) Second, the replicated partition function is averaged over the ensemble Eq. (1.4) At this stage, we depart from Refs 12, 14, 16, 17, 19 and do exact averaging without appealing to a nonlinear $\sigma$-model. This is the most crucial step in our derivation: It allows us to retain control over the analyticity of the resulting expressions considered as a function of $n$, the number of replicas.

(iii) Third, the 'density-density' correlation function is recovered by means of the double limiting procedure 22

$$
\begin{aligned}
\left\langle\rho_{N}(E) \rho_{N}\left(E^{\prime}\right)\right\rangle & =\lim _{\gamma, \gamma^{\prime} \rightarrow 0} \lim _{n \rightarrow \pm 0} \frac{1}{(2 \pi n)^{2}} \\
& \times \partial_{\gamma} \partial_{\gamma^{\prime}}\left\langle Z_{\gamma, \gamma^{\prime}}^{n}\left(E, E^{\prime}\right)\right\rangle
\end{aligned}
$$

and found to follow Eqs. (1.1) in the spectrum bulk after appropriate rescaling of spectral variables. [The limits $n \rightarrow \pm 0$ correspond to the fermionic and the bosonic replicas, respectively.]

The paper is organized as follows. In the auxiliary Section II, the notion of the product statistics is introduced, and a technique to average the product statistics is presented. With minor modifications, this section closely follows the recent paper by Tracy and Widom 23 whose impact we acknowledge. The results of Section II, represented in the form of the Grassmann functional integrals, are used in Section III where the fluctuation formulas Eqs. (1.1) are obtained. Conclusions are presented in Section IV.

\section{PRODUCT STATISTICS IN RANDOM MATRIX THEORY}

Let us introduce the notion of the product statistics $F$ defined on the eigenvalues $\{\lambda\}$ of $N \times N$ random matrix
$\mathbf{H}$ of appropriate symmetry:

$$
F\left(\lambda_{1}, \ldots, \lambda_{N}\right)=\prod_{k=1}^{N} f\left(\lambda_{k}\right)
$$

Here, $f(\lambda)$ is an arbitrary function of the variable $\lambda$ such that the integral $\int d \lambda \lambda^{j} f(\lambda) e^{-\beta V(\lambda)}$ exists for positive integers $j$.

This section aims to establish exact formulas for the average of the product statistics

$$
\langle F\rangle=\left\langle\prod_{k=1}^{N} f\left(\lambda_{k}\right)\right\rangle
$$

over the distribution function Eq. (1.4) for all three symmetry classes $\beta=1,2$ and 4 . We notice that along with utility of these formulas for computing the average of the replicated partition function, they may find applications in evaluating the average of other product statistics-encountered in description of a number of observables 2425 in mesoscopic physics.

\section{A. Unitary symmetry: $\beta=2$}

Due to invariance of the distribution function $P_{N}[\mathbf{H}]$ at $\beta=2$ under the unitary transformation $\mathrm{U}(N)$, the average $\langle F\rangle$ can be written down as $N$-fold integral

$$
\langle F\rangle \propto \int \mathcal{D} \hat{\Lambda} e^{-2 \operatorname{tr} V(\hat{\Lambda})}\left|\Delta_{N}(\hat{\Lambda})\right|^{2} \operatorname{det}[f(\hat{\Lambda})]
$$

where the proper normalization is to be restored on the later stage. Here, the diagonal matrix $\hat{\Lambda}=\operatorname{diag}\left(\lambda_{1}, \ldots, \lambda_{N}\right)$, the integration measure $\mathcal{D} \hat{\Lambda}=$ $\prod_{m=1}^{N} d \lambda_{m}$, and $\Delta_{N}(\hat{\Lambda})=\prod_{1 \leq i<j \leq N}\left(\lambda_{i}-\lambda_{j}\right)$ is the Vandermonde determinant.

Integration in Eq. (2.3) can be performed if we notice that the Vandermonde determinant $\Delta_{N}(\hat{\Lambda})=$ $\operatorname{det}\left[\lambda_{i}^{j-1}\right] \propto \operatorname{det}\left[P_{j-1}\left(\lambda_{i}\right)\right]$, where $P_{j}(\lambda)$ is a set of polynomials that we choose to be orthonormal with respect to the measure $e^{-2 V(\lambda)} d \lambda$. In terms of the 'wave functions' $\varphi_{j}(\lambda)=e^{-V(\lambda)} P_{j}(\lambda)$ this orthonormality is expressed as

$$
\int d \lambda \varphi_{i}(\lambda) \varphi_{j}(\lambda)=\delta_{i j}
$$

The integral Eq. (2.3) can be brought to the form

$$
\int\left(\prod_{m=1}^{N} d \lambda_{m} f\left(\lambda_{m}\right)\right) \operatorname{det}\left[\varphi_{j-1}\left(\lambda_{i}\right)\right]_{1 \leq i, j \leq N} \operatorname{det}\left[\varphi_{j-1}\left(\lambda_{i}\right)\right]_{1 \leq i, j \leq N}
$$


Integrating the product of two determinants in accordance with the formula 26

$$
\int\left(\prod_{m=1}^{N} d \lambda_{m}\right) \operatorname{det}\left[u_{j}\left(\lambda_{i}\right)\right]_{1 \leq i, j \leq N} \operatorname{det}\left[w_{j}\left(\lambda_{i}\right)\right]_{1 \leq i, j \leq N}=N ! \operatorname{det}\left(\int d \lambda u_{i}(\lambda) w_{j}(\lambda)\right)_{1 \leq i, j \leq N}
$$

that is proven via the Laplace expansion of the two determinants, we reduce Eq. (2.5) to

$$
\langle F\rangle \equiv \operatorname{det}\left(\int d \lambda f(\lambda) \varphi_{i-1}(\lambda) \varphi_{j-1}(\lambda)\right)_{1 \leq i, j \leq N}
$$

that enjoys a correct normalization owing to Eq. 2.4 and constitutes the first preliminary result to be used in the following Section.

\section{B. Symplectic symmetry: $\beta=4$}

In the case of the symplectic symmetry, $\operatorname{Sp}(N)$, the average $\langle F\rangle$ is given by

$$
\langle F\rangle \propto \int \mathcal{D} \hat{\Lambda} e^{-4 \operatorname{tr} V(\hat{\Lambda})}\left|\Delta_{N}(\hat{\Lambda})\right|^{4} \operatorname{det}[f(\hat{\Lambda})]
$$

where the correct normalization is to be found yet. Despite of appearance of $\left|\Delta_{N}(\hat{\Lambda})\right|^{4}$, that reflects the selfdual quaternion nature of the matrix $\mathbf{H}$ for $\beta=4$ in Eq.
(1.4), the exact integration in $\mathrm{Eq}_{-}(2.8)$ is still possible. First, we make use of the identity 27

$$
\begin{aligned}
\left|\Delta_{N}(\hat{\Lambda})\right|^{4} & =\operatorname{det}\left[\lambda_{i}^{j-1}, j \lambda_{i}^{j-1}\right] \\
& =\operatorname{det}\left[P_{j-1}\left(\lambda_{i}\right), P_{j-1}^{\prime}\left(\lambda_{i}\right)\right]_{1 \leq i \leq N, 1 \leq j \leq 2 N}
\end{aligned}
$$

to rewrite Eq. (2.8) as

$$
\begin{aligned}
& \int\left(\prod_{m=1}^{N} d \lambda_{m} \mathrm{e}^{-4 V\left(\lambda_{m}\right)} f\left(\lambda_{m}\right)\right) \\
& \times \operatorname{det}\left[P_{j-1}\left(\lambda_{i}\right), P_{j-1}^{\prime}\left(\lambda_{i}\right)\right]_{1 \leq i \leq N, 1 \leq j \leq 2 N}
\end{aligned}
$$

In both equations above, $P_{j}(\lambda)$ is a set of polynomials which we choose to be orthonormal with respect to the measure $e^{-4 V(\lambda)} d \lambda$. Second, theintegration in Eq. (2.10) can be performed via the analog 28 of Eq. (2.6) that reads

$$
\int\left(\prod_{m=1}^{N} d \lambda_{m}\right) \operatorname{det}\left[u_{j}\left(\lambda_{i}\right), w_{j}\left(\lambda_{i}\right)\right]_{1 \leq i \leq N, 1 \leq j \leq 2 N}=(2 N) ! \operatorname{pf}\left(\int d \lambda\left[u_{i}(\lambda) w_{j}(\lambda)-u_{j}(\lambda) w_{i}(\lambda)\right]\right)_{1 \leq i, j \leq 2 N}
$$

Here, the notation 'pf' stands for the pfaffiane9 [its square is determinant]. The identity Eq. 2.11) enables us to simplify Eq. 2.10 to

$$
\operatorname{pf}\left(\int d \lambda f(\lambda)\left[\varphi_{i-1}(\lambda) \varphi_{j-1}^{\prime}(\lambda)-\varphi_{j-1}(\lambda) \varphi_{i-1}^{\prime}(\lambda)\right]\right)_{1 \leq i, j \leq 2 N}
$$

where irrelevant overall constant has been omitted, and the 'wave functions' $\varphi_{j}(\lambda)=e^{-2 V(\lambda)} P_{j}(\lambda)$ obeying the orthonormality relation in the form of Eq. 2.4 have been introduced. Combining the above results together and properly restoring the normalization, we derive the following exact pfaffian representation for the averaged product statistics:

$$
\langle F\rangle \equiv \frac{\operatorname{pf}\left(\int d \lambda f(\lambda)\left[\varphi_{i-1}(\lambda) \varphi_{j-1}^{\prime}(\lambda)-\varphi_{j-1}(\lambda) \varphi_{i-1}^{\prime}(\lambda)\right]\right)_{1 \leq i, j \leq 2 N}}{\operatorname{pf}\left(\int d \lambda\left[\varphi_{i-1}(\lambda) \varphi_{j-1}^{\prime}(\lambda)-\varphi_{j-1}(\lambda) \varphi_{i-1}^{\prime}(\lambda)\right]\right)_{1 \leq i, j \leq 2 N}}
$$

Equation (2.13) is the second preliminary result needed in what follows.

\section{Orthogonal symmetry: $\beta=1$}

For $\beta=1$, the pfaffian representation for the averaged product statistics similar to Eq. (2.13) can also be obtained. To avoid unnecessary complications, we choose the real symmetric matrix $\mathbf{H}$ to be of an even dimension, $2 N \times 2 N$, so that the upper index in the products in Eqs. 2.1) and (2.2) is equal to $2 N$. Then, due to the $\mathrm{O}(2 N)$ invariance of the distribution function $P_{2 N}[\mathbf{H}]$, we encounter the following $2 N$-fold integral

$$
\langle F\rangle \propto \int \mathcal{D} \hat{\Lambda} e^{-\operatorname{tr} V(\hat{\Lambda})}\left|\Delta_{2 N}(\hat{\Lambda})\right| \operatorname{det}[f(\hat{\Lambda})]
$$

It is useful to reduce Eq. 2.14 to the form 29 


$$
\begin{aligned}
(2 N) ! & \int_{\lambda_{1} \leq \ldots \leq \lambda_{2 N}}\left(\prod_{m=1}^{2 N} d \lambda_{m} e^{-V\left(\lambda_{m}\right)} f\left(\lambda_{m}\right)\right) \\
& \times \prod_{1 \leq i<j \leq 2 N}\left(\lambda_{j}-\lambda_{i}\right)
\end{aligned}
$$

where the integration runs over the ordered domain $\lambda_{1} \leq \ldots \leq \lambda_{2 N}$, and therefore the modulus of the
Vandermonde determinant has been relaxed. Recalling that the Vandermonde determinant is proportional to $\operatorname{det}\left[P_{j-1}\left(\lambda_{i}\right)\right]$ with $1 \leq i, j \leq 2 N$ and choosing $P_{j}(\lambda)$ to be the polynomial orthonormal with respect to the measure $e^{-V(\lambda)} d \lambda$, we are able to perform integration in Eq. (2.15) by means of the identity 28

$$
\int_{\lambda_{1} \leq \ldots \leq \lambda_{2 N}}\left(\prod_{m=1}^{2 N} d \lambda_{m}\right) \operatorname{det}\left[u_{j}\left(\lambda_{i}\right)\right]_{1 \leq i, j \leq 2 N}=\operatorname{pf}\left(\int d \lambda \int d \lambda^{\prime} \operatorname{sgn}\left(\lambda^{\prime}-\lambda\right) u_{i}(\lambda) u_{j}\left(\lambda^{\prime}\right)\right)_{1 \leq i, j \leq 2 N},
$$

where $\operatorname{sgn}(\lambda)=\lambda /|\lambda|$. The integration results in

$$
\operatorname{pf}\left(\int d \lambda \int d \lambda^{\prime} \epsilon\left(\lambda-\lambda^{\prime}\right) f(\lambda) f\left(\lambda^{\prime}\right) \varphi_{i-1}(\lambda) \varphi_{j-1}\left(\lambda^{\prime}\right)\right)_{1 \leq i, j \leq 2 N},
$$

where irrelevant overall constant has been suppressed, and the 'wave functions' $\varphi_{j}(\lambda)=e^{-V(\lambda) / 2} P_{j}(\lambda)$ obeying the orthonormality relation in the form of Eq. 2.4) have appeared. Also, to meet the standard notations of Refs 21,23, we have introduced

$$
\epsilon(\lambda)=\frac{1}{2} \operatorname{sgn}(\lambda)
$$

Restoring the proper normalization, one arrives at the third preliminary result:

$$
\langle F\rangle \equiv \frac{\operatorname{pf}\left(\iint d \lambda d \lambda^{\prime} f(\lambda) f\left(\lambda^{\prime}\right) \epsilon\left(\lambda-\lambda^{\prime}\right) \varphi_{i-1}(\lambda) \varphi_{j-1}\left(\lambda^{\prime}\right)\right)_{1 \leq i, j \leq 2 N}}{\operatorname{pf}\left(\iint d \lambda d \lambda^{\prime} \epsilon\left(\lambda-\lambda^{\prime}\right) \varphi_{i-1}(\lambda) \varphi_{j-1}\left(\lambda^{\prime}\right)\right)_{1 \leq i, j \leq 2 N}}
$$

Notice that Eqs. (2.7), (2.13) and 2.19) are exact and refer to an arbitrary product statistics $F$ that does not affect the convergence of the integrals under the signs of 'det' and 'pf'.

\section{EIGENLEVEL CORRELATIONS FROM THE REPLICA METHOD}

\section{A. Unitary symmetry: $\beta=2$}

Exact determinantal expression for the averaged replicated partition function $\left\langle Z_{\gamma, \gamma^{\prime}}^{n}\left(E, E^{\prime}\right)\right\rangle$ in the case of the unitary symmetry is readily obtained from Eq. 2.7) upon specifying

$$
f(\lambda)=\left[(E-\lambda)^{2}+\gamma^{2}\right]^{n}\left[\left(E^{\prime}-\lambda\right)^{2}+\gamma^{\prime 2}\right]^{n},
$$

see Eq. (1.5). It should he noticed that, contrary to the $\sigma$-model representation 12.14.17.19] [see, also, Eqs. (1.2) and (1.3)], our determinantal form is especially useful to perform the replica limit $n \rightarrow \pm 0$, with +0 and -0 corresponding to the fermionic and the bosonic replicas, respectively. However, Eq. (2.7) as it stands, is not convenient for the large $-N$ analysis of the 'density-density' correlation function. For this reason, we put Eq. (2.7) into equivalent form of the functional integral over the scalar Grassmann field

$$
\psi(\lambda)=\sum_{k=1}^{N} \chi_{k} \varphi_{k-1}(\lambda)
$$

with the measure

$$
D[\bar{\psi}, \psi]=\prod_{k=1}^{N} d \chi_{k}^{*} d \chi_{k}
$$

where $\left\{\chi_{k}\right\}$ are $N$ Grassmann complex variables and $\bar{\psi}$ denotes the complex conjugate. One can verify 30 that the choice Eq. (3.2) enables us to rewrite Eq. (2.7) in the form of the functional integral

$$
\langle F\rangle \equiv \int D[\bar{\psi}, \psi] e^{\iint d x d y \bar{\psi}(x) K_{N}(x, y) f(y) \psi(y)}
$$

that solely involves the scalar kernel21

$$
K_{N}(x, y)=\sum_{k=0}^{N-1} \varphi_{k}(x) \varphi_{k}(y)=K_{N}(y, x)
$$

obeying the integral relationship

$$
\int d z K_{N}(x, z) K_{N}(z, y)=K_{N}(x, y) .
$$

In turn, the universal properties of the scatar - kernel $K_{N}(x, y)$ in the large $-N$ limit are well studied 3132. 
Substituting Eq. (3.1) into Eq. (3.4), differentiating the latter with respect to $\gamma$ and $\gamma^{\prime}$ and computing the limits $n \rightarrow \pm 0$ and $\gamma, \gamma^{\prime} \rightarrow 0$ one gets access to the 'density-density' correlation function in accordance with Eq. (1.6). Since we are interested in the correlations in the energy range where $E-E^{\prime}$ may take the values of order of the mean level spacing $\Delta_{N}$, we introduce the scaled variables $S=E / \Delta_{N}$ and $S^{\prime}=E^{\prime} / \Delta_{N}$, and restrict ourselves to the vicinity of the center of the eigen- value support, where $S, S^{\prime} \sim \mathcal{O}\left(N^{0}\right)$. In the large- $N$ limit, the rescaled Eq. (1.6) is then reduced to

$$
\begin{aligned}
\left\langle\rho(S) \rho\left(S^{\prime}\right)\right\rangle & =\Delta_{N}^{2} \lim _{\gamma, \gamma^{\prime} \rightarrow 0} \lim _{n \rightarrow \pm 0} \frac{1}{(2 \pi n)^{2}} \\
& \times \partial_{\gamma} \partial_{\gamma^{\prime}}\left\langle Z_{\gamma, \gamma^{\prime}}^{n}\left(S \Delta_{N}, S^{\prime} \Delta_{N}\right)\right\rangle .
\end{aligned}
$$

Straightforward calculations based on Eqs. (3.7) and (3.4) with $f(\lambda)$ given by Eq. (3.1) yield

$$
\begin{aligned}
\left\langle\rho(S) \rho\left(S^{\prime}\right)\right\rangle & =\delta\left(S-S^{\prime}\right) \Delta_{N}^{2} \int d \sigma K_{N}\left(\sigma \Delta_{N}, S \Delta_{N}\right)\left\langle\left\langle\bar{\psi}\left(\sigma \Delta_{N}\right) \psi\left(S \Delta_{N}\right)\right\rangle\right\rangle \\
& +\Delta_{N}^{4} \iint d \sigma d \sigma^{\prime} K_{N}\left(\sigma \Delta_{N}, S \Delta_{N}\right)\left\langle\left\langle\bar{\psi}\left(\sigma \Delta_{N}\right) \psi\left(S \Delta_{N}\right) \bar{\psi}\left(\sigma^{\prime} \Delta_{N}\right) \psi\left(S^{\prime} \Delta_{N}\right)\right\rangle\right\rangle K_{N}\left(\sigma^{\prime} \Delta_{N}, S^{\prime} \Delta_{N}\right)
\end{aligned}
$$

where the double angular brackets $\langle\langle\ldots\rangle\rangle$ stand for the Gaussian average

$$
\langle\langle\ldots\rangle\rangle=\int D[\bar{\psi}, \psi](\ldots) e^{\Delta_{N}^{2} \iint d \sigma d \sigma^{\prime} \bar{\psi}\left(\sigma \Delta_{N}\right) K_{N}\left(\sigma \Delta_{N}, \sigma^{\prime} \Delta_{N}\right) \psi\left(\sigma^{\prime} \Delta_{N}\right)} .
$$

In Eq. (3.8), $\langle\langle\bar{\psi} \psi\rangle\rangle$ and $\langle\langle\bar{\psi} \psi \bar{\psi} \psi\rangle\rangle$ may be computed via the Wick theorem33:

$$
\begin{aligned}
\left\langle\left\langle\bar{\psi}\left(\sigma_{1} \Delta_{N}\right) \psi\left(\sigma_{2} \Delta_{N}\right)\right\rangle\right\rangle & =K_{N}\left(\sigma_{1} \Delta_{N}, \sigma_{2} \Delta_{N}\right) \\
\left\langle\left\langle\bar{\psi}\left(\sigma_{1} \Delta_{N}\right) \psi\left(\sigma_{2} \Delta_{N}\right) \bar{\psi}\left(\sigma_{3} \Delta_{N}\right) \psi\left(\sigma_{4} \Delta_{N}\right)\right\rangle\right\rangle & =K_{N}\left(\sigma_{1} \Delta_{N}, \sigma_{2} \Delta_{N}\right) K_{N}\left(\sigma_{3} \Delta_{N}, \sigma_{4} \Delta_{N}\right) \\
& -K_{N}\left(\sigma_{1} \Delta_{N}, \sigma_{4} \Delta_{N}\right) K_{N}\left(\sigma_{3} \Delta_{N}, \sigma_{2} \Delta_{N}\right) .
\end{aligned}
$$

As far as we are concerned with the spectrum bulk, one has $\Delta_{N} K_{N}\left(S \Delta_{N}, S^{\prime} \Delta_{N}\right)=K(s)$ with

$$
K(s)=\frac{\sin (\pi s)}{\pi s},
$$

$s=\left|S-S^{\prime}\right|$, by the universality arguments31,32, 21. This equation holds for the strong confinement potential 34 only.

Combining now Eqs. (3.8), (3.10) and (3.11), we come down to

$$
\left\langle\rho(S) \rho\left(S^{\prime}\right)\right\rangle=1-K^{2}(s)+\delta(s)
$$

which is clearly identical to Eq. (1.1b). This is the central result of the subsection.
It must be stressed that, by derivation, Eq. 3.12) is valid in the bulk of the spectrum without the restriction $s$ schemes 14 17. The self-correlation of energy levels is also reproduced.

\section{B. Symplectic symmetry: $\beta=4$}

For technical reasons dictated by the pfaffian structure of Eq. 2.13) derived for $\beta=4$, it is useful to deal with $\left\langle Z_{\gamma, \gamma^{\prime}}^{n}\left(E, E^{\prime}\right)\right\rangle^{2}$ instead of $\left\langle Z_{\gamma, \gamma^{\prime}}^{n}\left(E, E^{\prime}\right)\right\rangle$. In such a case, the 'density-density' correlation function is seen to be generated by the replica limit

$$
\left\langle\rho_{N}(E) \rho_{N}\left(E^{\prime}\right)\right\rangle=\frac{1}{2} \lim _{\gamma, \gamma^{\prime} \rightarrow 0} \lim _{n \rightarrow \pm 0} \frac{1}{(2 \pi n)^{2}}\left[\partial_{\gamma} \partial_{\gamma^{\prime}}\left\langle Z_{\gamma, \gamma^{\prime}}^{n}\left(E, E^{\prime}\right)\right\rangle^{2}-\frac{1}{2} \partial_{\gamma}\left\langle Z_{\gamma, \gamma^{\prime}}^{n}\left(E, E^{\prime}\right)\right\rangle^{2} \partial_{\gamma^{\prime}}\left\langle Z_{\gamma, \gamma^{\prime}}^{n}\left(E, E^{\prime}\right)\right\rangle^{2}\right]
$$

with $\left\langle Z_{\gamma, \gamma^{\prime}}^{n}\left(E, E^{\prime}\right)\right\rangle^{2}$ being the ratio of two determinants, Eq. (2.13); the function $f(\lambda)$ is given by Eq. (3.1).

To facilitate the large $-N$ analysis, we rewrite the squared Eq. 2.13) in the equivalent form of the ratio of two Grassmann functional integrals:

$$
\langle F\rangle^{2} \equiv \frac{\int D[\bar{\Psi}, \Psi] e^{\iint d x d y \bar{\Psi}(x) \hat{K}_{N}(x, y) f(y) \Psi(y)}}{\int D[\bar{\Psi}, \Psi] e^{\iint d x d y \bar{\Psi}(x) \hat{K}_{N}(x, y) \Psi(y)}} .
$$

Here, $\Psi(x)$ is the two-component Grassmann field

$$
\Psi(x)=\sum_{k=1}^{2 N} \chi_{k} \phi_{k-1}(x)=\sum_{k=1}^{2 N} \chi_{k}\left(\begin{array}{c}
\varphi_{k-1}^{\prime}(x) \\
\varphi_{k-1}(x)
\end{array}\right)
$$

and $\bar{\Psi}(x)=\Psi^{\dagger}(x) L$, with

$$
L=\left(\begin{array}{cc}
0 & -1 \\
1 & 0
\end{array}\right) \text {. }
$$


Also, the integration measure is

$$
D[\bar{\Psi}, \Psi]=\prod_{k=1}^{2 N} d \chi_{k}^{*} d \chi_{k}
$$

The choice Eq. (3.15) naturally leads to appearance of the $2 \times 2$ matrix kernel

$$
\hat{K}_{N}(x, y)=\left(\begin{array}{ll}
K_{N}^{11}(x, y) & K_{N}^{12}(x, y) \\
K_{N}^{21}(x, y) & K_{N}^{22}(x, y)
\end{array}\right)
$$

which is a close analog of the scalar kernel $K_{N}(x, y)$, Eq. (3.5), arising in random matrix ensembles with $\mathrm{U}(N)$ symmetry. In terms of the wave functions $\varphi_{k}(x)$ of Eq. (2.13), the $(\alpha, \beta)$ entry of the matrix kernel is given by

$$
\hat{K}_{N}^{\alpha \beta}(x, y)=(-1)^{\beta-1} \sum_{j, k=1}^{2 N} \varphi_{j-1}^{(2-\alpha)}(x) \mu_{j k} \varphi_{k-1}^{(\beta-1)}(y),
$$

$[\alpha, \beta=1,2]$, so that the integral identity

$$
\int d z \hat{K}_{N}(x, z) \hat{K}_{N}(z, y)=\hat{K}_{N}(x, y)
$$

holds. Here, $\varphi_{k}^{(\alpha)}(x)$ denotes the derivative of $\alpha$-th order with respect to $x$, and $\mu_{j k}=\left(M^{-1}\right)_{j k}$, where $2 N \times 2 N$ antisymmetric matrix $M$ has the entries
$M_{j k}=\int d \lambda\left[\varphi_{j-1}(\lambda) \varphi_{k-1}^{\prime}(\lambda)-\varphi_{k-1}(\lambda) \varphi_{j-1}^{\prime}(\lambda)\right]$,

$j, k \in(1, \ldots, 2 N)$. Equation (3.19) coincides with the one found for the first time by Tracy and Widom 23. As is the case of $\mathrm{U}(N)$ symmetry, the large- $N$ hehavior of the matrix kernel $\hat{K}_{N}$ is known in detail 21.35.36.

The representation Eq. (3.14) is a bit less trivial as compared to Eq. (3.4). To verify the former, one should observe that $\phi_{k}(x)$ in Eq. (3.15) is the right eigenvector of the matrix kernel $\hat{K}_{N}(x, y)$ :

$$
\int d y \hat{K}_{N}(x, y) \phi_{k}(y)=\phi_{k}(x)
$$

while $\bar{\phi}_{k}(x)=\phi_{k}^{\dagger}(x) L$ is its left eigenvector:

$$
\int d x \bar{\phi}_{k}(x) \hat{K}_{N}(x, y)=\bar{\phi}_{k}(y)
$$

with $k \in(0, \ldots, 2 N-1)$.

In what follows we are interested in the 'densitydensity' correlation function in rescaled energy variables $S=E / \Delta_{N}$ and $S^{\prime}=E^{\prime} / \Delta_{N}$, when the large $-N$ limit of Eq. (3.13) transforms to

$$
\begin{aligned}
\left\langle\rho(S) \rho\left(S^{\prime}\right)\right\rangle & =\frac{\Delta_{N}^{2}}{2} \lim _{\gamma, \gamma^{\prime} \rightarrow 0} \lim _{n \rightarrow \pm 0} \frac{1}{(2 \pi n)^{2}} \\
& \times\left[\partial_{\gamma} \partial_{\gamma^{\prime}}\left\langle Z_{\gamma, \gamma^{\prime}}^{n}\left(S \Delta_{N}, S^{\prime} \Delta_{N}\right)\right\rangle^{2}-\frac{1}{2} \partial_{\gamma}\left\langle Z_{\gamma, \gamma^{\prime}}^{n}\left(S \Delta_{N}, S^{\prime} \Delta_{N}\right)\right\rangle^{2} \partial_{\gamma^{\prime}}\left\langle Z_{\gamma, \gamma^{\prime}}^{n}\left(S \Delta_{N}, S^{\prime} \Delta_{N}\right)\right\rangle^{2}\right] .
\end{aligned}
$$

Substituting Eq. (3.14) with $f(\lambda)$ given by Eq. (3.1) into Eq. (3.24) leads us to the following representation:

$$
\begin{aligned}
\left\langle\rho(S) \rho\left(S^{\prime}\right)\right\rangle & =\frac{1}{2}\left[\delta\left(S-S^{\prime}\right) \Delta_{N}^{2} \int d \sigma \sum_{\alpha, \beta=1}^{2} \hat{K}_{N}^{\alpha \beta}\left(\sigma \Delta_{N}, S \Delta_{N}\right)\left\langle\left\langle\bar{\Psi}^{\alpha}\left(\sigma \Delta_{N}\right) \Psi^{\beta}\left(S \Delta_{N}\right)\right\rangle\right\rangle\right. \\
& +\Delta_{N}^{4} \iint d \sigma d \sigma^{\prime} \sum_{\alpha, \beta=1}^{2} \sum_{\gamma, \delta=1}^{2} \hat{K}_{N}^{\alpha \beta}\left(\sigma \Delta_{N}, S \Delta_{N}\right) \hat{K}_{N}^{\gamma \delta}\left(\sigma^{\prime} \Delta_{N}, S^{\prime} \Delta_{N}\right) \\
& \times\left(\left\langle\left\langle\bar{\Psi}^{\alpha}\left(\sigma \Delta_{N}\right) \Psi^{\beta}\left(S \Delta_{N}\right) \bar{\Psi}^{\gamma}\left(\sigma^{\prime} \Delta_{N}\right) \Psi^{\delta}\left(S^{\prime} \Delta_{N}\right)\right\rangle\right\rangle\right. \\
& \left.\left.-\frac{1}{2}\left\langle\left\langle\bar{\Psi}^{\alpha}\left(\sigma \Delta_{N}\right) \Psi^{\beta}\left(S \Delta_{N}\right)\right\rangle\right\rangle\left\langle\left\langle\bar{\Psi}^{\gamma}\left(\sigma^{\prime} \Delta_{N}\right) \Psi^{\delta}\left(S^{\prime} \Delta_{N}\right)\right\rangle\right\rangle\right)\right] .
\end{aligned}
$$

Here, the double angular brackets $\langle\langle\ldots\rangle\rangle$ denote the Gaussian average

$$
\langle\langle\ldots\rangle\rangle=\frac{\int D[\bar{\Psi}, \Psi](\ldots) e^{\Delta_{N}^{2} \iint d \sigma d \sigma^{\prime} \bar{\Psi}\left(\sigma \Delta_{N}\right) \hat{K}_{N}\left(\sigma \Delta_{N}, \sigma^{\prime} \Delta_{N}\right) \Psi\left(\sigma^{\prime} \Delta_{N}\right)}}{\int D[\bar{\Psi}, \Psi] e^{\Delta_{N}^{2} \iint d \sigma d \sigma^{\prime} \bar{\Psi}\left(\sigma \Delta_{N}\right) \hat{K}_{N}\left(\sigma \Delta_{N}, \sigma^{\prime} \Delta_{N}\right) \Psi\left(\sigma^{\prime} \Delta_{N}\right)}} .
$$

The averages of the type $\left\langle\left\langle\bar{\Psi}^{\alpha} \Psi^{\beta}\right\rangle\right\rangle$ and $\left\langle\left\langle\bar{\Psi}^{\alpha} \Psi^{\beta} \bar{\Psi}^{\gamma} \Psi^{\delta}\right\rangle\right\rangle$ in Eq. (3.25) are evaluated by means of the Wick theorem [see Appendix for details]; below we display the result:

$$
\begin{aligned}
\left\langle\left\langle\bar{\Psi}^{\alpha}\left(\sigma_{1} \Delta_{N}\right) \Psi^{\beta}\left(\sigma_{2} \Delta_{N}\right)\right\rangle\right\rangle & =\hat{K}_{N}^{\beta \alpha}\left(\sigma_{2} \Delta_{N}, \sigma_{1} \Delta_{N}\right) \\
\left\langle\left\langle\bar{\Psi}^{\alpha}\left(\sigma_{1} \Delta_{N}\right) \Psi^{\beta}\left(\sigma_{2} \Delta_{N}\right) \bar{\Psi}^{\gamma}\left(\sigma_{3} \Delta_{N}\right) \Psi^{\delta}\left(\sigma_{4} \Delta_{N}\right)\right\rangle\right\rangle & =\hat{K}_{N}^{\beta \alpha}\left(\sigma_{2} \Delta_{N}, \sigma_{1} \Delta_{N}\right) \hat{K}_{N}^{\delta \gamma}\left(\sigma_{4} \Delta_{N}, \sigma_{3} \Delta_{N}\right) \\
& -\hat{K}_{N}^{\delta \alpha}\left(\sigma_{4} \Delta_{N}, \sigma_{1} \Delta_{N}\right) \hat{K}_{N}^{\beta \gamma}\left(\sigma_{2} \Delta_{N}, \sigma_{3} \Delta_{N}\right) .
\end{aligned}
$$


Inserting Eqs. (3.27) into Eq. (3.25), and making use of Eq. (3.20), one obtains:

$$
\begin{aligned}
\left\langle\rho(S) \rho\left(S^{\prime}\right)\right\rangle & =\frac{1}{2}\left[\delta\left(S-S^{\prime}\right) \Delta_{N} \operatorname{tr}\left[\hat{K}_{N}\left(S \Delta_{N}, S \Delta_{N}\right)\right]\right. \\
& \left.+\Delta_{N}^{2}\left(\frac{1}{2} \operatorname{tr}\left[\hat{K}_{N}\left(S \Delta_{N}, S \Delta_{N}\right)\right] \operatorname{tr}\left[\hat{K}_{N}\left(S^{\prime} \Delta_{N}, S^{\prime} \Delta_{N}\right)\right]-\operatorname{tr}\left[\hat{K}_{N}\left(S \Delta_{N}, S^{\prime} \Delta_{N}\right) \hat{K}_{N}\left(S^{\prime} \Delta_{N}, S \Delta_{N}\right)\right]\right)\right] .
\end{aligned}
$$

Here, the trace 'tr' acts in the $2 \times 2$ space of the matrix kernel $\hat{K}_{N}$.

In the large- $N$ limit, the kernel $\hat{K}_{N}\left(S \Delta_{N}, S^{\prime} \Delta_{N}\right)$ taken in the bulk of the spectrum which is of our primary interest] obeys the universal law 35. 36,21

$$
\begin{aligned}
\Delta_{N} \hat{K}_{N}^{11}\left(S \Delta_{N}, S^{\prime} \Delta_{N}\right) & =\Delta_{N} \hat{K}_{N}^{22}\left(S \Delta_{N}, S^{\prime} \Delta_{N}\right) \\
& =K(s) \\
\Delta_{N}^{2} \hat{K}_{N}^{12}\left(S \Delta_{N}, S^{\prime} \Delta_{N}\right) & =K^{\prime}(s), \\
\hat{K}_{N}^{21}\left(S \Delta_{N}, S^{\prime} \Delta_{N}\right) & =\int_{0}^{s} d t K(t),
\end{aligned}
$$

provided the confinement potential is strong 4 . Here, $s=\left|S-S^{\prime}\right|$, and

$$
K(s)=\frac{\sin (2 \pi s)}{2 \pi s} .
$$

Combining Eqs. (3.28), (3.29) and (3.30), we come down to

$$
\left\langle\rho(S) \rho\left(S^{\prime}\right)\right\rangle=1-K^{2}(s)+\frac{d K(s)}{d s} \int_{0}^{s} d t K(t)+\delta(s) .
$$

Equation (3.31), derived from the replicas, is seen to identically coincide with Eq. (1.10) for arbitrary s. It represents the main result of the subsection.

\section{Orthogonal symmetry: $\beta=1$}

As is the case of the symplectic symmetry, it is useful to deal with $\left\langle Z_{\gamma, \gamma^{\prime}}^{n}\left(E, E^{\prime}\right)\right\rangle^{2}$, so that the 'densitydensity' correlation function is defined by the replica limit Eq. (3.13) with $N$ being replaced by $2 N$, and with $\left\langle Z_{\gamma, \gamma^{\prime}}^{n}\left(E, E^{\prime}\right)\right\rangle^{2}$ given by the ratio of two determinants as follows from Eq. (2.19); the function $f(\lambda)$ is specified by Eq. (3.1).

In order to simplify the large $-N$ analysis, we have to express $\left\langle Z_{\gamma, \gamma^{\prime}}^{n}\left(E, E^{\prime}\right)\right\rangle^{2}$ in terms of the Grassmann functional integrals. One can verify that the squared averaged partition function admits the representation

$$
\langle F\rangle^{2} \equiv \frac{\int D[\bar{\Psi}, \Psi] e \iint d x d y \bar{\Psi}(x) f(x)\left[\hat{K}_{2 N}(x, y)-\hat{Q}_{2 N}(x, y)\right] f(y) \Psi(y)}{\int D[\bar{\Psi}, \Psi] e^{\iint d x d y \bar{\Psi}(x)\left[\hat{K}_{2 N}(x, y)-\hat{Q}_{2 N}(x, y)\right] \Psi(y)}} .
$$

Here, the integration measure $D[\bar{\Psi}, \Psi]$ is given by Eq. (3.17). The two-component Grassmann field $\Psi$ is defined as

$$
\Psi(x)=\sum_{k=1}^{2 N} \chi_{k} \phi_{k-1}(x)=\sum_{k=1}^{2 N} \chi_{k}\left(\begin{array}{c}
\varphi_{k-1}(x) \\
{\left[\hat{\epsilon} \varphi_{k-1}\right](x)}
\end{array}\right),
$$

with $\bar{\Psi}(x)=\Psi^{\dagger}(x) L ; 2 \times 2$ matrix $L$ is given by Eq. (3.16). Also, $\hat{\epsilon}$ denotes the integral operator associated with Eq. (2.18); it acts on some function $g(x)$ in accordance with the rule

$$
[\hat{\epsilon} g](x)=\int d y \epsilon(x-y) g(y)
$$

provided the integral is convergent.

The representation Eq. (3.32) involves the $2 \times 2$ matrices $\hat{Q}_{2 N}$ and $\hat{K}_{2 N}$ which are related to each other as

$$
\hat{Q}_{2 N}(x, y)=\hat{K}_{2 N}(x, y)+\left(\begin{array}{cc}
0 & 0 \\
\epsilon(x-y) & 0
\end{array}\right)
$$

with

$$
\hat{Q}_{2 N}^{\alpha \beta}(x, y)=(-1)^{\beta} \sum_{j, k=1}^{2 N}\left[\hat{\epsilon}^{\alpha-1} \varphi_{j-1}\right](x) \mu_{j k}\left[\hat{\epsilon}^{2-\beta} \varphi_{k-1}\right](y),
$$

$[\alpha, \beta=1,2]$. Here, the matrix $\mu_{j k}=\left(M^{-1}\right)_{j k}$, where $2 N \times 2 N$ antisymmetric matrix $M$ has the entries

$$
M_{j k}=\iint d x d y \varphi_{j-1}(x) \epsilon(x-y) \varphi_{k-1}(y)
$$

$j, k \in(1, \ldots, 2 N)$.

Let us point out that the matrix $\hat{K}_{2 N}(x, y)$ is nothing but the $2 \times 2$ matrix kerne 23 describing energy level correlations in random matrix ensembles with orthogonal symmetry. However, contrary to Eq. (3.20), the kernel $\hat{K}_{2 N}(x, y)$ obeys the more complicated integral identity

$$
\int d z \hat{K}_{2 N}(x, z) \hat{K}_{2 N}(z, y)=\hat{K}_{2 N}(x, y)
$$




$$
-\frac{1}{2}\left[\hat{K}_{2 N}(x, y), \Lambda\right],
$$

where $[\ldots, \ldots]$ stands for commutator, and the matrix $\Lambda=\operatorname{diag}(+1,-1)$. The auxiliary matrix $\hat{Q}_{2 N}(x, y)$ satisfies

$$
\int d z \hat{Q}_{2 N}(x, z) \hat{Q}_{2 N}(z, y)=2 \hat{Q}_{2 N}(x, y) .
$$

An advantage of the representation Eq. (3.32) is in the fact that the Gaussian averages of the type $\left\langle\left\langle\bar{\Psi}^{\alpha} \Psi^{\beta}\right\rangle\right\rangle$ and
$\left\langle\left\langle\bar{\Psi}^{\alpha} \Psi^{\beta} \bar{\Psi}^{\gamma} \Psi^{\delta}\right\rangle\right\rangle$ arising when implementing the replica limit may easily be expressed in terms of the large $-N$ matrix kernel $\hat{K}_{2 N}$; the universal properties of the latter for the strong level confinement 34 are well studied $35,36,21$.

Restricting ourselves to the spectrum bulk, the 'density-density' correlation function in rescaled variables $S=E / \Delta_{2 N}$ and $S^{\prime}=E^{\prime} / \Delta_{2 N}$ is given by the replica limit Eq. (3.24). Substituting there Eq. (3.32) with $f(\lambda)$ specified by Eq. (3.1) and taking into account Eq. (3.35), we obtain

$$
\begin{aligned}
\left\langle\rho(S) \rho\left(S^{\prime}\right)\right\rangle & =\frac{\Delta_{2 N}^{2}}{2}\left\{\epsilon\left(S-S^{\prime}\right)\left[\left\langle\left\langle\bar{\Psi}^{2}\left(S^{\prime} \Delta_{2 N}\right) \Psi^{1}\left(S \Delta_{2 N}\right)\right\rangle\right\rangle-\left\langle\left\langle\bar{\Psi}^{2}\left(S \Delta_{2 N}\right) \Psi^{1}\left(S^{\prime} \Delta_{2 N}\right)\right\rangle\right\rangle\right]\right. \\
& +\delta\left(S-S^{\prime}\right) \int d \sigma \epsilon(S-\sigma)\left[\left\langle\left\langle\bar{\Psi}^{2}\left(\sigma \Delta_{2 N}\right) \Psi^{1}\left(S \Delta_{2 N}\right)\right\rangle\right\rangle-\left\langle\left\langle\bar{\Psi}^{2}\left(S \Delta_{2 N}\right) \Psi^{1}\left(\sigma \Delta_{2 N}\right)\right\rangle\right\rangle\right] \\
& +\Delta_{2 N}^{2} \iint d \sigma d \sigma^{\prime} \epsilon\left(S^{\prime}-\sigma\right) \epsilon\left(S-\sigma^{\prime}\right)\left[\left\langle\left\langle\bar{\Psi}^{2}\left(S^{\prime} \Delta_{2 N}\right) \Psi^{1}\left(\sigma \Delta_{2 N}\right) \bar{\Psi}^{2}\left(S \Delta_{2 N}\right) \Psi^{1}\left(\sigma^{\prime} \Delta_{2 N}\right)\right\rangle\right\rangle\right. \\
& +\left\langle\left\langle\bar{\Psi}^{2}\left(\sigma \Delta_{2 N}\right) \Psi^{1}\left(S^{\prime} \Delta_{2 N}\right) \bar{\Psi}^{2}\left(\sigma^{\prime} \Delta_{2 N}\right) \Psi^{1}\left(S \Delta_{2 N}\right)\right\rangle\right\rangle \\
& -\left\langle\left\langle\bar{\Psi}^{2}\left(S^{\prime} \Delta_{2 N}\right) \Psi^{1}\left(\sigma \Delta_{2 N}\right) \bar{\Psi}^{2}\left(\sigma^{\prime} \Delta_{2 N}\right) \Psi^{1}\left(S \Delta_{2 N}\right)\right\rangle\right\rangle \\
& -\left\langle\left\langle\bar{\Psi}^{2}\left(\sigma \Delta_{2 N}\right) \Psi^{1}\left(S^{\prime} \Delta_{2 N}\right) \bar{\Psi}^{2}\left(S \Delta_{2 N}\right) \Psi^{1}\left(\sigma^{\prime} \Delta_{2 N}\right)\right\rangle\right\rangle \\
& -\frac{1}{2}\left(\left\langle\left\langle\bar{\Psi}^{2}\left(S^{\prime} \Delta_{2 N}\right) \Psi^{1}\left(\sigma \Delta_{2 N}\right)\right\rangle\right\rangle-\left\langle\left\langle\bar{\Psi}^{2}\left(\sigma \Delta_{2 N}\right) \Psi^{1}\left(S^{\prime} \Delta_{2 N}\right)\right\rangle\right\rangle\right) \\
& \left.\left.\times\left(\left\langle\left\langle\bar{\Psi}^{2}\left(S \Delta_{2 N}\right) \Psi^{1}\left(\sigma^{\prime} \Delta_{2 N}\right)\right\rangle\right\rangle-\left\langle\left\langle\bar{\Psi}^{2}\left(\sigma^{\prime} \Delta_{2 N}\right) \Psi^{1}\left(S \Delta_{2 N}\right)\right\rangle\right\rangle\right)\right]\right\} .
\end{aligned}
$$

Here, the notation $\langle\langle\ldots\rangle\rangle$ stands for the Gaussian average

$$
\langle\langle\ldots\rangle\rangle=\frac{\int D[\bar{\Psi}, \Psi](\ldots) e^{\Delta_{2 N}^{2} \iint d \sigma d \sigma^{\prime} \bar{\Psi}\left(\sigma \Delta_{2 N}\right)\left[\hat{K}_{2 N}\left(\sigma \Delta_{2 N}, \sigma^{\prime} \Delta_{2 N}\right)-\hat{Q}_{2 N}\left(\sigma \Delta_{2 N}, \sigma^{\prime} \Delta_{2 N}\right)\right] \Psi\left(\sigma^{\prime} \Delta_{2 N}\right)}}{\int D[\bar{\Psi}, \Psi] e^{\Delta_{2 N}^{2} \iint d \sigma d \sigma^{\prime} \bar{\Psi}\left(\sigma \Delta_{2 N}\right)\left[\hat{K}_{2 N}\left(\sigma \Delta_{2 N}, \sigma^{\prime} \Delta_{2 N}\right)-\hat{Q}_{2 N}\left(\sigma \Delta_{2 N}, \sigma^{\prime} \Delta_{2 N}\right)\right] \Psi\left(\sigma^{\prime} \Delta_{2 N}\right)}} .
$$

For the averages $\langle\langle\ldots\rangle\rangle$ the Wick theorem holds [see Appendix for details], so that

$$
\begin{aligned}
\left\langle\left\langle\bar{\Psi}^{\alpha}\left(\sigma_{1} \Delta_{2 N}\right) \Psi^{\beta}\left(\sigma_{2} \Delta_{2 N}\right)\right\rangle\right\rangle & =-\hat{Q}_{2 N}^{\beta \alpha}\left(\sigma_{2} \Delta_{2 N}, \sigma_{1} \Delta_{2 N}\right) \\
\left\langle\left\langle\bar{\Psi}^{\alpha}\left(\sigma_{1} \Delta_{2 N}\right) \Psi^{\beta}\left(\sigma_{2} \Delta_{2 N}\right) \bar{\Psi}^{\gamma}\left(\sigma_{3} \Delta_{2 N}\right) \Psi^{\delta}\left(\sigma_{4} \Delta_{2 N}\right)\right\rangle\right\rangle & =\hat{Q}_{2 N}^{\beta \alpha}\left(\sigma_{2} \Delta_{2 N}, \sigma_{1} \Delta_{2 N}\right) \hat{Q}_{2 N}^{\delta \gamma}\left(\sigma_{4} \Delta_{2 N}, \sigma_{3} \Delta_{2 N}\right) \\
& -\hat{Q}_{2 N}^{\delta \alpha}\left(\sigma_{4} \Delta_{2 N}, \sigma_{1} \Delta_{2 N}\right) \hat{Q}_{2 N}^{\beta \gamma}\left(\sigma_{2} \Delta_{2 N}, \sigma_{3} \Delta_{2 N}\right) .
\end{aligned}
$$

Inserting Eqs. (3.42) into Eq. (3.40) and taking into account Eqs. (3.35) and (3.36), one derives after some algebra:

$$
\begin{aligned}
\left\langle\rho(S) \rho\left(S^{\prime}\right)\right\rangle & =\delta\left(S-S^{\prime}\right) \Delta_{2 N} \hat{K}_{2 N}^{11}\left(S \Delta_{2 N}, S \Delta_{2 N}\right)+\Delta_{2 N}^{2}\left[\hat{K}_{2 N}^{11}\left(S \Delta_{2 N}, S \Delta_{2 N}\right) \hat{K}_{2 N}^{11}\left(S^{\prime} \Delta_{2 N}, S^{\prime} \Delta_{2 N}\right)\right. \\
& \left.-\hat{K}_{2 N}^{11}\left(S \Delta_{2 N}, S^{\prime} \Delta_{2 N}\right) \hat{K}_{2 N}^{22}\left(S \Delta_{2 N}, S^{\prime} \Delta_{2 N}\right)+\hat{K}_{2 N}^{12}\left(S \Delta_{2 N}, S^{\prime} \Delta_{2 N}\right) \hat{K}_{2 N}^{21}\left(S \Delta_{2 N}, S^{\prime} \Delta_{2 N}\right)\right] .
\end{aligned}
$$

In the large- $N$ limit, the kernel $\hat{K}_{2 N}\left(S \Delta_{2 N}, S^{\prime} \Delta_{2 N}\right)$ taken in the bulk fo the spectrum is known to obey the universal laws 35.3621

$$
\begin{aligned}
\Delta_{2 N} \hat{K}_{2 N}^{11}\left(S \Delta_{2 N}, S^{\prime} \Delta_{2 N}\right) & =\Delta_{2 N} \hat{K}_{2 N}^{22}\left(S \Delta_{2 N}, S^{\prime} \Delta_{2 N}\right) \\
& =K(s), \\
\Delta_{2 N}^{2} \hat{K}_{2 N}^{12}\left(S \Delta_{2 N}, S^{\prime} \Delta_{2 N}\right) & =K^{\prime}(s), \\
\hat{K}_{2 N}^{21}\left(S \Delta_{2 N}, S^{\prime} \Delta_{2 N}\right) & =-\int_{s}^{+\infty} d t K(t),
\end{aligned}
$$

provided the confinement potential is strong 4 . Here, $s=\left|S-S^{\prime}\right|$, and

$$
K(s)=\frac{\sin (\pi s)}{\pi s} .
$$

Taking into account Eqs. (3.44) and (3.45), one concludes that Eq. (3.43) [which is valid for arbitrary $s$ ] is equivalent to 


$$
\left\langle\rho(S) \rho\left(S^{\prime}\right)\right\rangle=1-K^{2}(s)-\frac{d K(s)}{d s} \int_{s}^{+\infty} d t K(t)+\delta(s)
$$

that, in turn, is identical to Eq. (1.12) as one could already expect.

\section{CONCLUSIONS}

The computation presented above has been inspired by the recent paper 14 by Kamengy and Mézard, followed by the further developments16.17.19, where the problem of the fermionic replicamethod has been revisited. The main outcome of Refs. 14.16.17 consists of assertion that the replica method in its fermionic $\sigma$-model elaboration, which relies on a selected set of causal saddle points 19 , is capable of reproducing the nonperturbative results for the spectral statistics, both in Gaussian invariant ensemblest 17 in the thermodynamic limit, and in $d-$ dimensional disordered conductors with finite Thouless conductance, $g \gg 1$, albeit in the large- $s$ domain, $s \gg 1$.

In this paper we examined the replica method from a different point of view, considering the fermionic and the bosonic replicas on an equal footing. The crucial difference between the previous studies and the present consideration resides in using an alternative [to the $\sigma$-model approach] technique to average the replicated partition function over the ensemble of random matrices for all three Dyson symmetry classes. This technique generates such representations for the spectral observables which are especially suitable for taking the replica limit $n \rightarrow \pm 0$ in a straightforward way. Performing exact [large $-N$ ] transformations at each stage, we have derived the nonperturbative random-matrix-theory fluctuation formulas for the spectral correlation functions in arbitrary spectrum range, including the effect of self-correlation of the energy levels showing up in the $\delta$-functional contribution in Eqs. (3.12), (3.31) and (3.46). Let us notice that the $\delta$-functional contribution is not an academic issue: It is this $\delta$-function whose spreading 37 [in the presence of an external perturbation] determines such important characteristics of disordered system as distributions of level velocities 38 and level curvatures 39 .

Reproducing of exact fluctuation formulas for invariant random matrix ensembles at $\beta=1,2$ and 4 through the replica method comprises the main outcome of our study. It explicitly demonstrates that, at least in the context of invariant random matrix models, the replica method itself is free from internal subtleties and thus completely legitimate, provided one is able to control the analytic properties of the replicated partition function Eq. (1.5) as a function of the replica parameter $n$ after the averaging over realizations of the random matrix Hamiltonian $\mathbf{H}$. The availability of such a control in the $\sigma$-model-formalizations of the replica method is still an open issue.

\section{ACKNOWLEDGMENTS}

Numerous discussions with V. E. Kravtsov are appreciated with thanks.

\section{APPENDIX: EQUATIONS (3.27) AND (3.42)}

(i) Consider the average in the l.h.s. of Eq. (3.27a), in which, by definition,

$$
\begin{aligned}
\bar{\Psi}^{\alpha}(x) & =(-1)^{\alpha-1} \sum_{k=1}^{2 N} \chi_{k}^{*} \varphi_{k-1}^{(\alpha-1)}(x), \\
\Psi^{\beta}(y) & =\sum_{k=1}^{2 N} \chi_{k} \varphi_{k-1}^{(2-\beta)}(y),
\end{aligned}
$$

$[\alpha, \beta=1,2]$, see Eqs. (3.15) and (3.16). To compute the required average given by Eq. (3.26), we write

$$
\left\langle\left\langle\bar{\Psi}^{\alpha}\left(\sigma_{1} \Delta_{N}\right) \Psi^{\beta}\left(\sigma_{2} \Delta_{N}\right)\right\rangle\right\rangle=(-1)^{\alpha-1} \sum_{j, k=1}^{2 N}\left\langle\chi_{j}^{*} \chi_{k}\right\rangle \varphi_{j-1}^{(\alpha-1)}\left(\sigma_{1} \Delta_{N}\right) \varphi_{k-1}^{(2-\beta)}\left(\sigma_{2} \Delta_{N}\right),
$$

where

$$
\left\langle\chi_{j}^{*} \chi_{k}\right\rangle=\frac{\int\left(\prod_{m=1}^{2 N} d \chi_{m}^{*} d \chi_{m}\right) \chi_{j}^{*} \chi_{k} e^{\sum_{p, q=1}^{2 N} \chi_{p}^{*} M_{p q} \chi_{q}}}{\int\left(\prod_{m=1}^{2 N} d \chi_{m}^{*} d \chi_{m}\right) e^{\sum_{p, q=1}^{2 N} \chi_{p}^{*} M_{p q} \chi_{q}}},
$$

and $M_{p q}$ is specified by Eq. (3.21). Since the Grassmann integral Eq. (A.4) equals $\left(M^{-1}\right)_{k j} \equiv \mu_{k j}$, one derives from Eq. (A.3): 


$$
\left\langle\left\langle\bar{\Psi}^{\alpha}\left(\sigma_{1} \Delta_{N}\right) \Psi^{\beta}\left(\sigma_{2} \Delta_{N}\right)\right\rangle\right\rangle=(-1)^{\alpha-1} \sum_{j, k=1}^{2 N} \varphi_{j-1}^{(\alpha-1)}\left(\sigma_{1} \Delta_{N}\right) \mu_{k j} \varphi_{k-1}^{(2-\beta)}\left(\sigma_{2} \Delta_{N}\right)=\hat{K}_{N}^{\beta \alpha}\left(\sigma_{2} \Delta_{N}, \sigma_{1} \Delta_{N}\right) .
$$

This completes the proof of Eq. (3.27a).

(ii) Consider the average in the l.h.s. of Eq. (3.27b). Making use of Eqs. (A.1) and (A.2), we rewrite the average as

$$
\begin{aligned}
\left\langle\left\langle\bar{\Psi}^{\alpha}\left(\sigma_{1} \Delta_{N}\right) \Psi^{\beta}\left(\sigma_{2} \Delta_{N}\right) \bar{\Psi}^{\gamma}\left(\sigma_{3} \Delta_{N}\right) \Psi^{\delta}\left(\sigma_{4} \Delta_{N}\right)\right\rangle\right\rangle & =(-1)^{\alpha+\gamma} \sum_{r, p, q, s=1}^{2 N}\left\langle\chi_{r}^{*} \chi_{p} \chi_{q}^{*} \chi_{s}\right\rangle \\
& \times \varphi_{r-1}^{(\alpha-1)}\left(\sigma_{1} \Delta_{N}\right) \varphi_{p-1}^{(2-\beta)}\left(\sigma_{2} \Delta_{N}\right) \varphi_{q-1}^{(\gamma-1)}\left(\sigma_{3} \Delta_{N}\right) \varphi_{s-1}^{(2-\delta)}\left(\sigma_{4} \Delta_{N}\right),
\end{aligned}
$$

where

$$
\left\langle\chi_{r}^{*} \chi_{p} \chi_{q}^{*} \chi_{s}\right\rangle=\frac{\int\left(\prod_{m=1}^{2 N} d \chi_{m}^{*} d \chi_{m}\right) \chi_{r}^{*} \chi_{p} \chi_{q}^{*} \chi_{s} e^{\sum_{p, q=1}^{2 N} \chi_{p}^{*} M_{p q} \chi_{q}}}{\int\left(\prod_{m=1}^{2 N} d \chi_{m}^{*} d \chi_{m}\right) e^{\sum_{p, q=1}^{2 N} \chi_{p}^{*} M_{p q} \chi_{q}}} \equiv \mu_{p r} \mu_{s q}-\mu_{s r} \mu_{p q}
$$

Inserting this result into Eq. (A.6) and appealing to Eq. (3.19), we arrive at the r.h.s. of Eq. (3.27b). This completes the proof.

(iii) Consider the average in the l.h.s. of Eq. (3.42a in notations of Eq. (3.41). By definition,

$$
\begin{aligned}
\bar{\Psi}^{\alpha}(x) & =(-1)^{\alpha-1} \sum_{k=1}^{2 N} \chi_{k}^{*}\left[\hat{\epsilon}^{2-\alpha} \varphi_{k-1}\right](x), \\
\Psi^{\beta}(y) & =\sum_{k=1}^{2 N} \chi_{k}\left[\hat{\epsilon}^{\beta-1} \varphi_{k-1}\right](y)
\end{aligned}
$$

$[\alpha, \beta=1,2]$, see Eq. (3.33). The required average is

$$
\left\langle\left\langle\bar{\Psi}^{\alpha}\left(\sigma_{1} \Delta_{2 N}\right) \Psi^{\beta}\left(\sigma_{2} \Delta_{2 N}\right)\right\rangle\right\rangle=(-1)^{\alpha-1} \sum_{j, k=1}^{2 N}\left\langle\chi_{j}^{*} \chi_{k}\right\rangle\left[\hat{\epsilon}^{2-\alpha} \varphi_{j-1}\right]\left(\sigma_{1} \Delta_{2 N}\right)\left[\hat{\epsilon}^{\beta-1} \varphi_{k-1}\right]\left(\sigma_{2} \Delta_{2 N}\right),
$$

where $\left\langle\chi_{j}^{*} \chi_{k}\right\rangle$ is given by Eq. (A.4) with $M_{p q}$ defined by Eq. (3.37). We then conclude that $\left\langle\chi_{j}^{*} \chi_{k}\right\rangle$ equals $\left(M^{-1}\right)_{k j}=$ $\mu_{k j}$, so that

$$
\left\langle\left\langle\bar{\Psi}^{\alpha}\left(\sigma_{1} \Delta_{2 N}\right) \Psi^{\beta}\left(\sigma_{2} \Delta_{2 N}\right)\right\rangle\right\rangle=(-1)^{\alpha-1} \sum_{j, k=1}^{2 N}\left[\hat{\epsilon}^{2-\alpha} \varphi_{j-1}\right]\left(\sigma_{1} \Delta_{2 N}\right) \mu_{k j}\left[\hat{\epsilon}^{\beta-1} \varphi_{k-1}\right]\left(\sigma_{2} \Delta_{2 N}\right)=-\hat{Q}_{2 N}^{\beta \alpha}\left(\sigma_{2} \Delta_{2 N}, \sigma_{1} \Delta_{2 N}\right) .
$$

Here, we have used Eq. (3.36). This completes the proof of Eq. (3.42a).

The proof of Eq. (3.42b) is straightforward and goes along the lines of (ii) and (iii).

${ }^{1}$ S. F. Edwards and P. W. Anderson, J. Phys. F 5, 965 (1975).

${ }^{2}$ F. J. Wegner, Z. Phys. B 35, 207 (1979); L. Schäfer and F. J. Wegner, Z. Phys. B 38, 113 (1980).

${ }^{3}$ K. B. Efetov, A. I. Larkin, and D. E. Khmelnitskii, Sov. Phys. JETP 52, 568 (1980).

${ }^{4}$ K. B. Efetov, Sov. Phys. JETP 56, 467 (1982); K. B. Efetov, Adv. Phys. 32, 53 (1983).

${ }^{5}$ K. B. Efetov, Supersymmetry in Disorder and Chaos (Cambridge University Press, Cambridge, 1997).
${ }^{6}$ J. J. M. Verbaarschot, H. A. Weidenmüller, and M. R. Zirnbauer, Phys. Rep. 129, 367 (1985).

${ }^{7}$ E. P. Wigner, Proc. Cambridge Philos. Soc. 47, 790 (1951).

${ }^{8}$ L. P. Gorkov and G. M. Eliashberg, Sov. Phys. JETP 21, 940 (1965).

${ }^{9}$ A. M. Finkelstein, Sov. Phys. JETP 57, 97 (1983); Z. Phys. B 56, 189 (1984).

${ }^{10}$ J. J. M. Verbaarschot and M. R. Zirnbauer, Ann. Phys. (Leipzig) 158, 78 (1984).

11 B. L. Altshuler and B. I. Shklovskii, Sov. Phys. JETP 64, 127 (1986).

12 J. J. M. Verbaarschot and M. R. Zirnbauer, J. Phys. A 17, 
1093 (1985).

13 J. L. van Hemmen and R. G. Palmer, J. Phys. A 12, 563 (1979).

14 A. Kamenev and M. Mézard, J. Phys. A 32, 4373 (1999); eprint cond-mat/9901110.

${ }^{15}$ C. Itzykson and J. B. Zuber, J. Math. Phys. 21, 411 (1980).

16 A. Kamenev and M. Mézard, Phys. Rev. B 60, 3944 (1999); eprint cond-mat/9903001.

${ }^{17}$ I. V. Yurkevich and I. V. Lerner, Phys. Rev. B 60, 3955 (1999); eprint cond-mat/9903025 (1999);

18 A. V. Andreev and B. L. Altshuler, Phys. Rev. Lett. 75, 902 (1995).

19 M. R. Zirnbauer, eprint cond-mat/9903338 (1999).

${ }^{20}$ J. J. Duistermaat and G. Heckman, Inv. Math. 69, 259 (1982); Inv. Math. 72, 153 (1983).

${ }^{21}$ M. L. Mehta, Random Matrices (Academic Press, Boston, 1991).

${ }^{22}$ This formula is used for $\beta=2$. For technical reasons, at $\beta=1$ and $\beta=4$ we make use of Eq. (3.13).

${ }^{23}$ C. A. Tracy and H. Widom, J. Stat. Phys. 92, 809 (1998).

${ }^{24}$ H. Lee, L. S. Levitov, and A. Yu. Yakovets, Phys. Rev. B 51, 4079 (1995); K. A. Muttalib and Y. Chen, Int. J. Mod. Phys. B 10, 1999 (1996).

${ }^{25}$ A. V. Andreev and B. D. Simons, Phys. Rev. Lett. 75, 2304 (1995).

${ }^{26}$ C. Andréief, Mém. de la Soc. Sci., Bordeaux 2, 1 (1883).
${ }^{27}$ H. W. Segar, Messenger of Mathematics 22, 57 (1893).

${ }^{28}$ N. G. de Bruijn, J. Indian Math. Soc. 19, 133 (1955).

${ }^{29}$ M. L. Mehta, Matrix Theory: Selected Topics and Useful Results (Les Editions de Physique, 1989).

30 The simplest way to do so is to notice that $\varphi_{k}(x)$ is simultaneously the left and the right eigenfunction of the operator $K_{N}(x, y)$, i. e. $\int d y K_{N}(x, y) \varphi_{k}(y)=\varphi_{k}(x)$ and $\int d x \varphi_{k}(x) K_{N}(x, y)=\varphi_{k}(y)$ for $k \in(0, \ldots, N-1)$.

${ }^{31}$ E. Brézin and A. Zee, Nucl. Phys. B 402, 613 (1993).

${ }^{32}$ E. Kanzieper and V. Freilikher in: Waves in Diffuse $\mathrm{Me}$ dia, edited by J.-P. Fouque, NATO ASI, Ser. C, Vol. 531 (Kluwer, Dordrecht, 1999); E. Kanzieper and V. Freilikher, Phys. Rev. Lett. 78, 3806 (1997).

33 The identities Eqs. 3.10 ) can be proven along the lines of the Appendix where a bit more involved cases of symplectic and orthogonal symmetries are treated.

34 The confinement potentials $V(E)$ is said to be 'strong' if it increases at least as $|E|$ at infinity.

${ }^{35}$ H. Widom, J. Stat. Phys. 94, 347 (1999).

${ }^{36}$ M. Şener and J. J. M. Verbaarschot, Phys. Rev. Lett. 81, 248 (1998).

${ }^{37}$ V. E. Kravtsov and M. R. Zirnbauer, Phys. Rev. B 46, 4332 (1992).

${ }^{38}$ N. Taniguchi, A. Hashimoto, B. D. Simons, and B. L. Altshuler, Europhys. Lett. 27, 335 (1994).

${ }^{39}$ F. von Oppen, Phys. Rev. Lett. 73, 798 (1994). 